\title{
Le vieillissement de la population en milieu rural québécois
}

\author{
Majella Simard ${ }^{\mathrm{a}}$
}

RÉSUMÉ. Le vieillissement de la population représente un défi incontournable pour le Québec, en particulier pour les milieux ruraux, plus affectés par ce phénomène. L'objectif de cet article consiste à examiner la trajectoire évolutive du vieillissement au sein des localités rurales du Québec au cours de la période 1986-2016. Les résultats sont exposés à partir d'une analyse typologique à trois niveaux réunissant des localités qui présentent certaines affinités eu égard à l'évolution et à l'intensité du vieillissement. L'analyse est effectuée suivant une approche géographique et socioéconomique. Parmi les 62 localités rurales les plus enclines au vieillissement, la plupart présentent des signes de fragilité sur les plans démographique, géographique et économique, ce qui témoigne du caractère structurel des différents malaises auxquels elles font face. La combinaison de ces difficultés contribue à handicaper leur développement, en plus d'avoir des impacts négatifs sur leurs perspectives d'avenir, d'où la nécessité de mettre en place une politique d'aménagement du territoire et de développement régional qui va bien au-delà d’une stratégie tournée uniquement vers le vieillissement démographique.

\begin{abstract}
The aging population presents an unavoidable challenge for Quebec and, in particular, for rural areas, most influenced by this phenomenon. This article examines the evolutionary trajectory of aging in rural Quebec during the thirty-year period from 1986 to 2016. A three-level typological analysis of localities reveals certain patterns with regard to the evolution and intensity of aging, notably concerning geography and socio-economic factors. Of the 62 rural areas most prone to aging, most show signs of demographic, geographical and economic fragility, reflecting the complex structural nature of the challenges they meet. The combination of these difficulties hampers their current development and negatively impacts their future prospects in many ways, bence the need to implement new regional development and organizational policies that go beyond considering demographic aging as the only factor.
\end{abstract}

\section{Introduction}

Le vieillissement de la population, défini par l'augmentation de la proportion des personnes âgées de 65 ans ou plus au cours d'une période donnée, représente l'un des phénomènes les plus cruciaux de notre société (Dumont, 2018; Broussy, 2014). Conjugué à d'autres réalités démographiques, telles que l'émigration rurale et la dénatalité, le vieillissement, dont le rythme évolutif et l'intensité diffèrent considérablement d'un endroit à l'autre, contribue à la déstructuration des pyramides d'âges.

En milieu rural, le phénomène demeure encore peu étudié (Pagès, 2013). Il n'est pourtant pas sans conséquence en matière d'offre de services de proximité, dont l'infrastructure tend à s'effriter particulièrement au sein des plus petites localités éloignées des villes (Simard, à paraître). En outre, il est susceptible d'influer sur la répartition géographique des activités économiques, soit en favorisant l'essor d'une économie résidentielle au sein des milieux présentant de fortes aménités rurales ou se situant à proximité des villes, soit en fragilisant les localités dont l'économie repose sur l'extraction ou la transformation des ressources en raison de la pénurie de main-d'œuvre (Attias-Donfut, 2013; Blanchet, 2013; Devazies, 1999). Pour toutes ces raisons, il importe de bien cerner l'ampleur du phénomène dans le but de mieux orienter les politiques publiques en matière d'aménagement du territoire et de développement régional.

L'objectif de notre contribution consiste à examiner la trajectoire évolutive du vieillissement au sein des milieux ruraux du Québec au cours de la période 1986-2016 à partir de recensements de Statistique Canada. Sur le plan méthodologique,

a Professeur, département d'histoire et de géographie, Université de Moncton 
nous privilégions une analyse typologique à trois niveaux (localités fortement, moyennement et légèrement enclines au vieillissement) réunissant des localités qui présentent certaines affinités eu égard à l'évolution du vieillissement. Notre analyse est effectuée suivant une approche géographique et socioéconomique. Dès lors, les résultats sont présentés en considérant des variables telles que l'évolution de la population entre 1986 et 2016, le taux d'activité, le taux de chômage, la nature de l'économie et du revenu total, les niveaux et la composition du revenu, le taux de scolarisation ainsi que certaines caractéristiques liées au tissu de peuplement.

Notre article comporte trois parties. En premier lieu, nous exposons brièvement les principaux enjeux du vieillissement démographique. La deuxième partie porte plus spécifiquement sur la présentation de nos résultats à l'échelle des milieux ruraux. Finalement, nous clôturons notre analyse en évoquant, à grands traits, quelques éléments de synthèse et de discussion.

\section{Les enjeux du vieillissement au Québec}

Les données du recensement de 2016 de Statistique Canada sont on ne peut plus révélatrices. Après Terre-Neuve-et-Labrador $(10,6)^{1}$ et le NouveauBrunswick $(8,8)$, le Québec $(8,3)$ est la province canadienne qui, entre 1986 et 2016, a été la plus affectée par le vieillissement de sa population. Le phénomène affecte de manière particulière les localités rurales du Québec, c'est-à-dire les subdivisions de recensement dont la population est inférieure à 2500 habitants ( $n=720$ en 2016). En effet, comparativement à l'ensemble de la province $(8,3)$, la proportion d'ainnés résidant en milieu rural s'est accrue de 11,1 points de pourcentage entre 1986 et 2016. Cette proportion grimpe à 12,2 points de pourcentage dans le cas des localités de moins de 500 habitants (Simard, à paraitre). La figure 1 représente l'évolution de la proportion de personnes âgées au sein des localités rurales du Québec au cours de cette période de 30 ans.

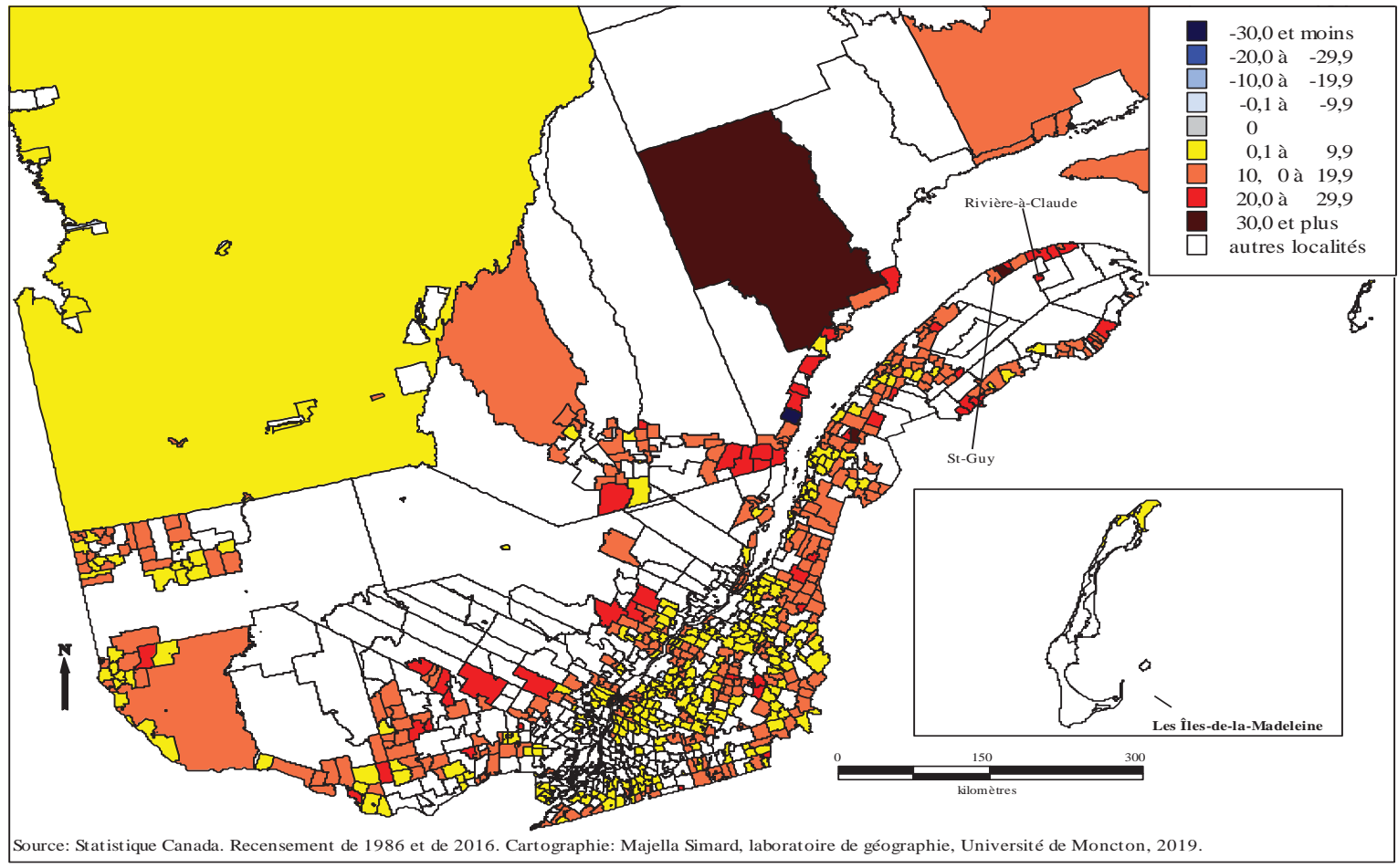

Figure 1 - Évolution de la proportion des personnes âgées de 65 ans ou plus dans les localités rurales du Québec entre 1986 et 2016 
De 1986 à 2016, la proportion d'aînés s'est accrue de 20 points de pourcentage ou plus dans 62 localités (voir tableau 1). Trois localités parmi celles-ci, en l'occurrence Rivière-à-Claude, Saint-Guy et Rivièreaux-Outardes, ont affiché une croissance de leur proportion de personnes âgées supérieure à 30 points de pourcentage. Nous les avons identifiées par leur toponyme à la figure 1. Mentionnons que cette même proportion a été inférieure à 10 points de pourcentage dans 326 subdivisions de recensement, alors que ce même indicateur a oscillé entre 10 et 19,9 points de pourcentage dans 323 localités (voir figure 1).
Par ailleurs, seulement 9 localités rurales ont connu une évolution négative de leur proportion d'aînés en l'espace de 30 ans, dont 4 abritent un fort contingent de population autochtone. Pour la plupart en croissance démographique, les 5 autres municipalités (Massueville, Saint-Célestin, Howick, Hemmingford et Les Escoumins) ont une économie qui repose surtout sur l'industrie touristique, ce qui les rend plus sujettes aux flux migratoires. En outre, la pratique d'activités de villégiature couplées à diverses initiatives mises en place pour attirer des jeunes expliquerait aussi le fait que ces localités ne sont pas enclines au vieillissement et, par conséquent, tendent à rajeunir.

\begin{tabular}{|c|c|c|c|c|c|}
\hline \multirow{2}{*}{$\begin{array}{l}\text { Variable/Évolution (en \%) de la proportion } \\
\text { de personnes âgées }\end{array}$} & Fortement & Moyennement & Légèrement & & \multirow{4}{*}{$\begin{array}{c}\text { Le } \\
\text { Québec }\end{array}$} \\
\hline & 20,0 et + & 10,0 à 19,9 & 0,1 à 9,9 & $-0,1$ et - & \\
\hline $\mathrm{n}$ & 62 & 323 & 326 & 9 & \\
\hline$\%$ & 8,6 & 44,8 & 45,2 & 1,4 & \\
\hline \multicolumn{6}{|l|}{ Variables démographiques } \\
\hline Population moyenne (2016) & 544 & 946 & 1086 & 738 & 7937 \\
\hline Évolution de la population (1986-2016) (n) & -9017 & 21646 & 36162 & 2728 & 1631900 \\
\hline Évolution de la population $(\%)$ & $-21,1$ & $-6,6$ & 11,4 & 69,8 & 25,0 \\
\hline $\begin{array}{l}\text { Nombre de localités en décroissance démographique } \\
\text { continue depuis } 1986\end{array}$ & 16 & 46 & 15 & 1 & 98 \\
\hline $\begin{array}{l}\text { Proportion de localités en décroissance démographique } \\
\text { continue depuis } 1986\end{array}$ & 25,8 & 14,2 & 4,6 & 11,1 & 9,6 \\
\hline Évolution des 0-24 ans (1986-2016) (n) & 11705 & 60895 & 24540 & -85 & 403110 \\
\hline Évolution des 0-24 ans (1986-2016) (\%) & $-67,2$ & $-46,9$ & $-18,9$ & $-3,5$ & 21,7 \\
\hline Évolution de la proportion des 0-24 ans (1986-2016) & $-23,8$ & $-17,1$ & $-11,1$ & $-26,9$ & $-13,5$ \\
\hline Évolution des 65 ans ou plus (1986-2016) (n) & 6830 & 40425 & 24280 & 150 & 1013620 \\
\hline Évolution des 65 ans ou plus (1986-2016) (\%) & 187,1 & 122,0 & 69,5 & 18,4 & 210,5 \\
\hline Évolution de la proportion des 65 ans ou plus (1986-2016) & 22,5 & 14,0 & 5,7 & $-6,3$ & 8,3 \\
\hline 0-24 ans en 1986 par rapport aux 30-54 ans en 2016 (n) & -8790 & 40535 & 16410 & -480 & 738740 \\
\hline $0-24$ ans en 1986 par rapport aux 30-54 ans en $2016(\%)$ & $-50,4$ & $-32,2$ & $-12,6$ & $-19,7$ & 41,1 \\
\hline \multicolumn{6}{|l|}{ Variables socioéconomiques } \\
\hline Taux de migrations pendulaires (2016) & 75,5 & 79,7 & 76,2 & 51,0 & 63,2 \\
\hline Taux d'activité de la population active (2016) & 45,7 & 55,5 & 63,2 & 67,1 & 64,1 \\
\hline Taux de chômage de la population active (2016) & 16,1 & 10,1 & 8,2 & 12,6 & 7,2 \\
\hline$\%$ des travailleurs affectés au secteur primaire (2016) & 12,7 & 12,6 & 13,5 & 6,8 & 2,5 \\
\hline$\%$ des travailleurs affectés au secteur secondaire (2016) & 22,7 & 23,2 & 22,6 & 13,2 & 17,1 \\
\hline \% des travailleurs affectés au secteur tertiaire (2016) & 64,6 & 64,2 & 63,9 & 80,0 & 80,4 \\
\hline $\begin{array}{l}\text { \% de la population active n'ayant pas complété une 9e année } \\
(2016)\end{array}$ & 30,6 & 25,7 & 23,9 & 29,7 & 15,1 \\
\hline $\begin{array}{l}\text { \% de la population active possédant un diplôme d'études } \\
\text { universitaires (2016) }\end{array}$ & 4,3 & 5,1 & 5,4 & 3,6 & 13,7 \\
\hline \multicolumn{6}{|l|}{ Variables liées au revenu } \\
\hline Revenu moyen des familles économiques* en \$ (2016) & 61527 & 66503 & 71932 & 73525 & 79378 \\
\hline $\begin{array}{l}\text { \% du revenu moyen des familles économiques par rapport à celui } \\
\text { du Québec (2016) }\end{array}$ & 77,5 & 83,8 & 90,6 & 92,6 & - \\
\hline \% du revenu provenant d'un emploi (2016) & 50,5 & 59,2 & 65,7 & 69,2 & 68,4 \\
\hline $\begin{array}{l}\text { \% du revenu provenant de transferts gouvernementaux } \\
(2016)\end{array}$ & 29,0 & 24,6 & 21,3 & 21,9 & 15,6 \\
\hline \% du revenu provenant d'une autre source (2016) & 20,5 & 16,2 & 13,0 & 8,9 & 16,0 \\
\hline$\%$ des familles économiques sous le seuil de faible revenu & 4,2 & 4,2 & 3,6 & 4,0 & 4,2 \\
\hline
\end{tabular}

* Pour Statistique Canada la famille économique renvoie à «un groupe de deux personnes ou plus habitant dans le même logement et apparentées par le sang, le mariage, l'union libre, l'adoption ou une relation de famille d'accueil. Un couple peut être de sexe opposé ou de même sexe. Par définition, toutes les personnes qui sont membres d'une famille de recensement sont également membres d'une famille économique » (Statisique Canada. Dictionnaire du recensement de 2016. En ligne : https://www12.statcan.gc.ca/census-recensement/2016/ref/dict/fam011-fra.cfm (consulté le 17 décembre 2019).

Tableau 1 - Principales caractéristiques socioéconomiques des localités rurales du Québec en fonction de l'intensité de leur vieillissement entre 1986 et 2016

Source : Statistique Canada, Recensements de 1986 et de 2016. Compilation et calculs de l'auteur. 


\begin{tabular}{|l|l|}
\hline Régions administratives & $\begin{array}{l}\text { Nbre de localités rurales } \\
\text { fortement vieillies }\end{array}$ \\
\hline Gaspésie & 12 \\
\hline Hautes-Laurentides & 12 \\
\hline Capitale-Nationale & 9 \\
\hline Chaudière-Appalaches & 7 \\
\hline Bas-Saint-Laurent & 6 \\
\hline Côte-Nord & 6 \\
\hline Saguenay-Lac-Saint-Jean & 5 \\
\hline Outaouais & 3 \\
\hline Abitibi-Témiscamingue & 1 \\
\hline Montérégie & 1 \\
\hline
\end{tabular}

Tableau 2-Nombre de localités rurales fortement enclines au vieillissement par régions administratives

\section{Situation du vieillissement au sein des milieux ruraux du Québec}

\subsection{Localités rurales les plus enclines au vieillissement}

De faible densité, la taille moyenne des 62 localités rurales les plus enclines au vieillissement est de 544 habitants. Elles ont tendance à se concentrer dans les régions périphériques du Québec (voir tableau 2). Avec 12 entrées, la Gaspésie occupe le peloton de tête ex aequo avec la région des Hautes-Laurentides.

\section{Évolution démographique}

De 1986 à 2016, la population de ces 62 localités est passée de 42732 personnes à 33715 , ce qui représente une diminution de $21,1 \%$. À titre indicatif, au cours de la même période, le Québec rural affichait un gain de 6516 habitants, soit une augmentation de $1 \%$. Parmi ces 62 localités, 6 ont perdu plus de la moitié de leur population en l'espace de 30 ans. Toutes se situent dans l'Est-duQuébec, ce qui illustre bien les défis titanesques auxquels certains milieux ruraux du Bas-SaintLaurent et de la Gaspésie font face sur le plan démographique. Il s'agit de Murdochville, de SaintGuy, de Saint-Jean-de-Cherbourg, de MontSaint-Pierre, de Sainte-Madeleine-de-la-RivièreMadeleine et de L'Ascension-de-Patapédia. De plus, 15 de ces 62 localités sont affectées par un dépeuplement structurel. En effet, 4 n'ont jamais cessé de perdre des effectifs depuis 1981, 3 depuis 1976 et 8 depuis 1971.

Néanmoins, les localités rurales fortement touchées par le vieillissement ne sont pas toutes affectées par le dépeuplement. C'est le cas en particulier de 16 d'entre elles, qui ont emprunté la trajectoire inverse. Les augmentations ont même été supérieures à $100 \%$ dans 5 de celles-ci, à savoir : LacSimon, Estérel, Rivière-aux-Outardes, Lac-Poulin et La Minerve.

\section{Évolution du nombre de jeunes}

La dénatalité et l'émigration ont entrainé, au sein des milieux ruraux fortement sujets au vieillissement, une forte décroissance du nombre de jeunes. En effet, alors qu'on dénombrait 17430 personnes de moins de 24 ans en 1986, ils n'étaient plus que 5725 en 2016, ce qui totalise une perte de 11705 personnes, soit une baisse de 67,2\%. Ni les localités moyennement ou légèrement enclines au vieillissement n'ont subi un fléchissement aussi prononcé de la cohorte des moins de 24 ans au cours de la période à l'étude.

La décroissance du nombre de jeunes apparait particulièrement problématique dans 23 de ces 62 localités rurales, lesquelles ont perdu plus des trois quarts des effectifs de cette cohorte en 30 ans. À Saint-Guy, la situation est telle qu'il ne restait plus aucun jeune en 2016, alors qu'ils n'étaient plus que 5 à Rivière-aux-Outardes et à Rivière-à-Claude. Toutefois, ce qui apparaît encore plus inquiétant est la diminution de la proportion de jeunes, un phénomène mieux connu sous l'appellation « vieillissement par le bas ». Cette dernière, au sein de ce même groupe, a régressé de 23,8 points de pourcentage en l'espace de 30 ans. En outre, en suivant les 0 à 24 ans en 1986, lesquels font partie de la cohorte des 30 à 54 ans en 2016, nous constatons une perte de 8790 personnes, ce qui représente une diminution de l'ordre de 50,4\%. 


\section{Enjeux économiques}

Les 62 localités les plus vieillies du Québec font aussi face à d'importants enjeux économiques. La précarité de leur économie se matérialise par un taux de chômage très élevé, lequel atteint 16,1 \% en 2016. Quant au taux d'activité, il s'établissait à $45,7 \%$, comparativement à $64,1 \%$ pour l'ensemble du Québec. Parmi ces 62 localités, 16 municipalités affichaient un taux inférieur à $40 \%$.

Les activités liées à l'exploitation des ressources composaient $12,7 \%$ de leur structure occupationnelle, contre $2,5 \%$ pour la province. Les secteurs secondaire et tertiaire retenaient respectivement $22,7 \%$ et $64,6 \%$ de la main-d'œuvre active locale, par rapport à $17,1 \%$ et à $80,4 \%$ pour la province. Les services accaparaient plus des trois quarts de la population active dans 15 de ces 62 municipalités. Bien que l'industrie touristique soit omniprésente dans la plupart de ces milieux et, de ce fait, explique la forte proportion d'emplois relevant du secteur tertiaire, pour plusieurs de ces municipalités, ce dernier constitue ni plus ni moins une sorte de refuge pour des individus qui ne sont pas en mesure d'occuper un emploi dans les autres branches de l'activité économique. C'est le cas notamment de Saint-Guy, de Sainte-Madeleine-de-la-RivièreMadeleine, de Matapédia et de Mont-Saint-Pierre, où les services composaient $85 \%$ ou plus de leur structure occupationnelle.

\section{Niveaux de scolarité}

La forte proportion de personnes âgées qu'on retrouve au sein des milieux plus enclins au vieillissement a pour corollaire la faiblesse de leur taux de scolarisation ainsi que la part considérable prise par les autres sources de revenus (dont celles inhérentes aux pensions de vieillesse) dans la composition du revenu total. Dans ces localités, en effet, la proportion d'individus n'ayant pas atteint le seuil de la $9^{\mathrm{e}}$ année se chiffrait à 30,6\%, par rapport à 15,1 \% pour le Québec. Les niveaux de scolarité les plus élevés se retrouvaient surtout dans les milieux à forte vocation touristique ou de villégiature, comme à Sainte-Pétronille et à Estérel, alors qu'à l'inverse les localités isolées des centres urbains, telles que Trinité-des-Monts, Saint-CharlesGarnier, Sainte-Marguerite, Saint-Médard et SaintVenant-de-Paquette, possédaient les taux les plus faibles.
Sources de revenus

Plus de $20 \%$ du revenu total au sein des localités comprises dans ce groupe provient d'une source autre qu'un emploi ou de transferts gouvernementaux, en comparaison à $16 \%$ pour la province. En 2016, le revenu moyen des milieux les plus vieillis s'établissait à 61527 \$, ce qui correspond à 77,5\% de celui du Québec. Certaines de ces municipalités, comme Sainte-Madeleine-de-la-Rivière-Madeleine, Sainte-Anne-du-Lac et Lac-du-Cerf, font partie des localités les plus pauvres du Québec, alors qu’à l'opposé, Abercorn et Sainte-Pétronille figurent parmi celles les plus riches. Enfin, pour cette même catégorie de milieux, la fréquence des ménages à faible revenu atteignait 4,2\%, ce qui équivaut à la moyenne québécoise. Par ailleurs, elle oscillait entre des extrêmes allant de 1,3\% dans le cas de SaintJulien à 8,3\% dans celui de Lac-du-Cerf.

\subsection{Localités rurrales moyennement enclines au vieillissement}

On dénombre 323 localités rurales dont l'augmentation de la proportion d'aînés a oscillé entre 10 et 19,9 points de pourcentage entre 1986 et 2016 (voir tableau 1). Leur taille moyenne s'établit à 946 habitants.

\section{Évolution démographique}

À l'instar du groupe précédent, la plupart de ces milieux $(\mathrm{n}=212)$ sont affectés par la décroissance démographique. En effet, de 327143 qu'elle était en 2016, leur population est passée à 305497 en 2016, ce qui représente une diminution de 6,6\%. Parmi les 212 localités en décroissance, 14 le sont depuis 1981, 3 depuis 1976 et 17 depuis 1971. Qui plus est, 40 localités ont perdu plus du tiers de leur population au cours de la période à l'étude.

À l'autre bout du spectre, 18 ont affiché une croissance démographique de l'ordre de $50 \%$ ou plus. Il s'agit de localités se situant à proximité d'une ville, à forte vocation touristique ou de villégiature. En l'espace de 30 ans, ces milieux se sont enrichis de 40225 personnes âgées de 65 ans ou plus, alors qu'ils ont perdu 60895 jeunes de moins de 24 ans. En moyenne, la proportion d'aînés s'est accrue de 14 points de pourcentage entre 1986 et 2016. Celleci a été supérieure à 18 points dans 32 localités, la plupart étant situées dans la région de ChaudièreAppalaches. 


\section{Évolution du nombre de jeunes}

Quant à la proportion de jeunes, elle a décru de 17,1 points. Aucune localité n'a connu une croissance de cet indicateur. Authier $(-7,1)$, Saint-Paulde-l'Île-aux-Noix (-9,1), Montebello $(-9,1)$ et Thorne $(-7,1)$ affichaient les baisses les plus faibles. À l'inverse, 22 localités ont enregistré une diminution de 25 points ou plus de leur proportion de jeunes. Celles-ci se retrouvent essentiellement sur la Côte-Nord, en Abitibi-Témiscamingue, en Gaspésie, dans le Bas-Saint-Laurent et dans Chaudière-Appalaches. Parmi ces localités, mentionnons Saint-Hilaire-de-Dorset (-41,5), Baie-Johan-Beetz $(-33,0)$, Rivière-Saint-Jean $(-32,1)$ et Saint-Jean-dela-Lande $(-30,7)$.

En suivant les 0 à 24 ans en 1986, lesquels font partie de la cohorte des 30 à 54 ans en 2016, seulement 40 localités ont subi une évolution positive de leurs effectifs. En valeur relative, les pertes, pour l'ensemble de cette catégorie, représentent une diminution de 40535 personnes. En valeur relative, il s'agit d'une décroissance de l'ordre de 32,2\%. Cette dernière a même été supérieure à $50 \%$ dans 59 localités, ce qui témoigne du fort courant d'émigration rurale auquel celles-ci sont assujetties. Saint-Omer-de-l'Islet $(-73,0)$, Rivière-Saint-Jean (-69,0), Grosses-Roches $(-68,2)$ et Petite-Vallée $(-68,0)$ font partie des milieux les plus affectés par ce phénomène.

\section{Enjeux économiques}

Sur le plan économique, plusieurs des localités appartenant à ce groupe souffrent de fragilité, et ce, bien que le taux d'activité moyen $(55,5 \%)$ soit supérieur à celui de la catégorie précédente (45,7\%; voir tableau 1). Par ailleurs, nous observons de très fortes inégalités au chapitre de l'emploi. Par exemple, des localités telles que Sainte-Hélène-deChester, Calixa-Lavallée, Matagami ou Natashquan ont un taux d'activité supérieur à $70 \%$. Certaines se situent près d'une ville, ce qui explique la vitalité de leur économie. Cette situation est d'ailleurs confirmée par des taux de migrations pendulaires très élevés. D’autres, souffrant d'encavement géographique, exercent le rôle de petits centres de services dans leur environnement immédiat. D'autres encore correspondent à des localités minières ou industrielles.
Il n'en demeure pas moins que le chômage, qui atteint une moyenne de 10,1\%, affecte la plupart des localités figurant au sein de cette catégorie, et ce, même si ce taux demeure moins élevé par rapport au groupe précédent (16,1\%). Le sous-emploi concerne plus spécifiquement 7 localités NotreDame-de-Lorette, Île d'Anticosti, Albertville, LacÉdouard, Saint-André-de-Restigouche, Rivière-auTonnerre et Franquelin), où le taux de chômage est supérieur à $30 \%$. La composition de la structure occupationnelle de ces milieux est pratiquement identique à celle de la catégorie précédente.

Néanmoins, la diversité caractérise les localités qui en font partie. Ainsi, dans ce groupe, le secteur primaire peut exercer un rôle majeur au sein de l'économie, comme c'est le cas à Notre-Dame-deLorette, à Lac-Chicobi, à Saint-Rémi-de-Tingwick et à Saint-Athanase. D'autres, comme SaintsMartyrs-Canadiens, Saint-Marc-du-Lac-Long, Lemieux ou Valcourt, disposent d'un solide appareil industriel.

Quant au secteur tertiaire, de trois choses l'une. D'abord, il sert de refuge à des travailleurs ne pouvant occuper un emploi dans d'autres sphères économiques. Nous pensons notamment à PetiteVallée, à Ristigouche-Partie-Sud-Est ou à SaintAndré-de-Restigouche. Dans d'autres cas, la forte proportion d'emplois relevant du secteur des services est imputable à la proximité d'une ville, comme c'est le cas pour Notre-Dame-du-Portage ou Pointe-à-la-Croix. Ailleurs, elle est attribuable au rôle de dispensateur de services qu'exercent ces localités au sein de leur milieu respectif. Les Escoumins et Nominingue s'inscrivent dans cette dernière situation.

Niveaux de scolarité

Un peu plus du quart de la population active n'avait pas atteint la 9e année en 2016, alors que 5,1 \% de celle-ci était détentrice d'un diplôme d'études universitaires. Une analyse fine des données permet de constater que les localités abritant une population faiblement scolarisée sont plutôt sujettes à la décroissance démographique, en plus de souffrir d'enclavement. S'inscrivent dans cette dernière situation Lac-Chicobi, Saint-Juste-de-Bretenières, Rivière-au-Tonnerre, Rivière-Ojima et Mont-SaintMichel. 


\section{Sources de revenus}

Le revenu moyen des familles économiques se chiffre à $66503 \$$, ce qui représente $83,8 \%$ de celui du Québec. Il oscille entre 47360 \$ (dans le cas de Grosses-Roches) et $123520 \$$ (dans celui de Vaudreuil-sur-le-Lac), illustrant de fortes disparités d'un milieu à l'autre. Le revenu d'emploi compose $59,2 \%$ du revenu total, contre $24,6 \%$ pour les transferts gouvernementaux et $16,2 \%$ pour ce qui est des autres sources de revenus.

\subsection{Localités rurrales légèrement enclines au vieillissement}

Les localités moins touchées par le vieillissement se composent d'un groupe de 326 municipalités, dont la population moyenne se chiffre à 1086 personnes (voir tableau 1).

\section{Évolution démographique}

Cette catégorie se distingue par une évolution démographique positive, ses effectifs ayant passé de 317910 à 354072 habitants entre 1986 et 2016, soit une croissance de $11,4 \%$. La population a même plus que doublé au sein de 27 d'entre elles, dont un bon nombre correspondent à des réserves autochtones.

La décroissance démographique est tout de même le lot de près de la moitié des localités comprises dans cette catégorie. Au nombre de 146, certains de ces milieux en déclin ont même perdu plus du tiers de leur population en 30 ans. C'est le cas de Schefferville, de Saint-François-Xavier-de-Viger, de Sainte-Jeanne-d'Arc, de Saint-Noël, de Champneuf et de Winneway. Le dépeuplement affecte de manière particulière 7 localités en décroissance continue depuis 1971, alors que 6 le sont depuis 1981.

De 34955 qu'ils étaient en 1986, le nombre d'aînés est passé à 59235 en 2016, soit une augmentation de $69,5 \%$. Néanmoins, certaines localités comme Lochaber-Partie-Ouest, Très-Saint-Rédempteur et Hatley sont particulièrement sujettes à la gérontocroissance, le nombre d'aînés ayant plus que triplé dans 14 localités.

\section{Évolution du nombre de jeunes}

Ce groupe est aussi celui où la diminution du nombre de jeunes $(-18,9 \%)$ a été la moins forte au cours de la période à l'étude (voir tableau 1). Près de 60 localités ont même connu une augmentation des 0 à 24 ans. Cette hausse s'est toutefois avérée insuffisante pour compenser les pertes encourues puisque seulement 3 localités, à savoir Kuujjuarapik, Belle-Rivière et Nemaska, n’ont pas été affectées par le vieillissement «par le bas ». En 1986, les moins de 24 ans totalisaient 129930 individus, alors que 113520 personnes faisaient partie de la cohorte des 30 à 54 ans en 2016, ce qui représente une décroissance de 16410 personnes. En valeur relative, il s'agit d'une diminution de $12,6 \%$. Si certaines localités sises en régions périphériques telles que Sainte-Jeanne-d'Arc, Saint-Cléophas, Portage-du-Fort ou Champneuf semblent fortement concernées par l'émigration rurale, d'autres comme Lac-Sergent, Pointe-des-Cascades ou Montcalm attirent de jeunes ménages en raison de leur situation géographique privilégiée ou de la qualité de leur cadre de vie.

\section{Enjeux économiques}

Les localités moins sujettes au vieillissement présentent une situation économique nettement supérieure à celle de leurs homologues des groupes précédents. Plusieurs signes témoignent de cette situation. D'abord, le taux d'activité moyen s'établit à 63,2 \%, avec des extrêmes allant de 29,8 \% (LacSimon) à 85,2\% (Belle-Rivière). Les activités liées à l'exploitation des ressources accaparent 13,5\% de la population active, soit la proportion la plus élevée de notre typologie, contre $22,6 \%$ pour celles liées à la transformation. De son côté, le secteur tertiaire compose $63,9 \%$ de la structure occupationnelle. Cette plus forte vitalité économique a pour corollaire un taux de chômage moins élevé, celui-ci s'établissant à 8,2\%.

\section{Niveaux de scolarité}

Comparativement aux localités fortement ou moyennement enclines au vieillissement, celles les moins affectées par ce phénomène présentent des niveaux de scolarité plus élevés. Toutefois, à l'image de la très grande diversité qui caractérise l'espace rural québécois, les disparités, particulièrement au sein des mielleux ruraux appartenant à ce groupe, restent fort considérables. Par exemple, la 
proportion d'individus n'ayant pas atteint le seuil de la 9e année n'est que de $5 \%$ à Lac-Sergent comparativement à 55,8 \% dans le cas de La Romaine, la moyenne s'établissant à 23,9\% \% De même, la proportion d'individus qui possèdent une formation universitaire, dont la moyenne se chiffre à 5,4\%, oscille entre des extrêmes allant de 0 (dans le cas de 16 localités) à 27,7\% pour celle de Senneville.

\section{Sources de revenus}

Au chapitre du revenu, ce dernier se chiffre à 71932 \$, ce qui représente 90,6\% de celui de la province. Cette moyenne gomme toutefois de fortes disparités, dont les écarts varient entre 33451 \$, dans le cas de Lac-Simon, la localité la plus pauvre du Québec eu égard à cet indicateur, à 165547 \$, dans celui de Fermont, celle la plus riche. Le revenu total se décline comme suit: $65,7 \%$ proviennent d'un emploi, $21,3 \%$ de transferts gouvernementaux, alors que les autres catégories de revenus forment $13 \%$ de celui-ci. Enfin, cette catégorie dispose du plus bas pourcentage de familles sous le seuil de faible revenu, cet indicateur étant même inférieur à la moyenne québécoise.

\section{Conclusion}

Le vieillissement représente un phénomène démographique qui affecte de manière particulière les milieux ruraux du Québec. Bien qu’il soit présent pratiquement partout, le vieillissement se manifeste à des intensités et des rythmes évolutifs qui diffèrent selon les endroits. Par exemple, les localités les plus touchées par cette réalité présentent plusieurs dénominateurs communs. Ayant tendance à se situer majoritairement, mais non exclusivement au sein des régions périphériques du Québec, elles sont généralement de petite taille démographique, en plus d'être éloignées des principaux pôles urbains. Le fait qu'elles soient plus enclines à la décroissance démographique et au vieillissement « par le bas » entraîne une déstructuration de leurs pyramides d'âges.

Elles se caractérisent aussi par la fragilité de leur économie, laquelle se manifeste par un faible taux d'activité, par un taux de chômage élevé ainsi que par une forte proportion d'emplois issus du secteur primaire. Dans bien des cas, elles abritent une population moins scolarisée, comparativement aux autres catégories de milieux ruraux, et à faible niveau de revenu.

Dès lors, le vieillissement se combine à une déprise spatiale et économique qui hypothèquent le devenir de ces milieux. En conséquence, la concentration de ces carences structurelles appelle à des interventions spécifiques de la part de l'État afin de les redynamiser, mais surtout d'améliorer la qualité de vie des personnes (tant des aînés que des plus jeunes) qui y résident. Ces interventions doivent se tourner à la fois vers l'aménagement du territoire, le développement local et régional ainsi que la démographie.

En ce qui concerne plus spécifiquement ce dernier aspect, l'élaboration d'une vigoureuse politique démographique gravitant autour de l'accroissement des naissances (et donc vers le renforcement des politiques familiales), du rapatriement des jeunes, d'un meilleur contrôle des flux migratoires et de l'attraction d'immigrants reposant sur une stratégie de régionalisation de l'immigration apparaît incontournable.

Sur le plan de l'aménagement du territoire, il importe de favoriser une meilleure occupation de l'espace rural en misant sur le renforcement de petits centres de services régionaux, de manière à consolider la structure de peuplement des localités sises au sein des régions périphériques du Québec, lesquelles se caractérisent généralement par la faiblesse de leur armature urbaine.

Enfin, en ce qui concerne le développement régional, la création d'emplois stables et bien rémunérés représente une piste à explorer afin d'encourager le maintien des jeunes au sein de leur région d'appartenance, ce qui permettrait de ralentir les effets du vieillissement.

Bref, si l'on vise la réduction des inégalités spatiales et l'équité territoriale comme cadre d'intervention, les différents acteurs (au premier chef, les instances gouvernementales supérieures) sont conviés à repenser la politique d'aménagement et de développement territorial qui prévaut depuis les 50 dernières années. Leurs actions doivent être orientées en considérant la diversité des situations et des trajectoires démographiques qui caractérisent l'espace rural québécois. 


\section{NOTES}

1 Le chiffre entre parenthèses indique l'augmentation de la proportion des personnes âgées de 65 ans ou plus entre 1986 et 2016. Il est exprimé en points de pourcentage.

\section{RÉFÉRENCES}

Attias-Donfut, C. (2013). Actions intergénérationnelles et développement durable en milieu rural. Gérontologie et société, 36(146), 117-129. https://doi.org/10.3917/gs.146.0117

Blanchet, M. (2013). Le vieillissement des campagnes : éléments d'introduction démographique et géographique. Gérontologie et société, 36(146), 21-38. https://doi.org/10.3917/gs.146.0021

Broussy, L. (2014). Dix mesures pour adapter la société française au vieillissement. Paris, France : Dunod.

Davezies, L. (1999). La territorialisation des personnes âgées : «l'or gris » et le « grey power ». Cabiers de l'Institut d'aménagement et d'urbanisme de la région d'île-de-France, 122, 193-197.

Dumont, G.-F. (2018). Le vieillissement dans le monde : conséquences géopolitiques. Vanguardia Dossier, 69, 123-127. Repéré à https:/ /halshs.archives-ouvertes.fr/halshs-01832382/document

Pagès, A. (2013). Des figures de pauvreté aux formes d'accompagnement de vieillir en milieu agricole et rural. Gérontologie et société, 36(146), 57-72. https://doi.org/10.3917/gs.146.0057

Simard, M. (à paraitre). Le vieillissement de la population et la décroissance démographique en milieu rural : un défi au développement des petites localités du Québec. Cabiers québécois de démographie.

Statistique Canada. Recensement de 1986 et de 2016. Analyseur du recensement canadien. Faculté des Arts et des sciences, Université de Toronto. http://dc1.chass.utoronto.ca/census/index.html 